AperTO - Archivio Istituzionale Open Access dell'Università di Torino

\title{
Annealing temperature dependence of the 2223 phase volume fraction in the Bi-Sr-Ca-Cu-O system
}

\section{This is the author's manuscript}

Original Citation:

\section{Availability:}

This version is available http://hdl.handle.net/2318/9957

since 2017-07-24T15:32:40Z

Publisher:

Elsevier BV:PO Box 211, 1000 AE Amsterdam Netherlands:011 3120 4853757, 0113120 4853642, 011

Terms of use:

Open Access

Anyone can freely access the full text of works made available as "Open Access". Works made available under a Creative Commons license can be used according to the terms and conditions of said license. Use of all other works requires consent of the right holder (author or publisher) if not exempted from copyright protection by the applicable law. 


\title{
Annealing temperature dependence of the 2223 phase volume fraction in the $\mathrm{Bi}-\mathrm{Sr}-\mathrm{Ca}-\mathrm{Cu}-\mathrm{O}$ system
}

\author{
C. Manfredotti ${ }^{a}$, M. Truccato ${ }^{a, *}$, G. Rinaudo ${ }^{a}$, D. Allasia ${ }^{a}$, P. Volpe ${ }^{b}$, \\ P. Benzi ${ }^{b}$, A. Agostino b \\ ${ }^{a}$ Dipartimento di Fisica Sperimentale, INFM-UdR Torino Università, Via P. Giuria, 1, I-10125 Torino, Italy \\ b Dipartimento di Chimica Generale ed Organica Applicata, C ${ }^{\text {so }}$ Massimo d'Azeglio 48, I-10125 Torino, Italy
}

Received 14 August 2000; received in revised form 9 October 2000; accepted 8 November 2000

\begin{abstract}
We have developed a reliable method based on AC magnetic susceptibility measurements to determine the $\mathrm{Bi}_{2} \mathrm{Sr}_{2} \mathrm{Ca}_{2} \mathrm{Cu}_{3} \mathrm{O}_{10+x}$ (2223) phase volume fraction and the average grain radius in multiphasic polycrystalline $\mathrm{Bi}-\mathrm{Sr}-\mathrm{Ca}-$ $\mathrm{Cu}-\mathrm{O}$ samples. Precision and applicability of the method are discussed and compared with those of the XRD peak integration procedure. The method is used to investigate the influence of the annealing temperature on the synthesis of the 2223 phase in air. The results show that, for a $120 \mathrm{~h}$ annealing time, $T=849^{\circ} \mathrm{C}$ is the most favourable among the investigated temperatures. Evidence is also given that higher temperatures promote the decomposition of the 2223 phase into the $\mathrm{Bi}_{2} \mathrm{Sr}_{2} \mathrm{CaCu}_{2} \mathrm{O}_{8+x}$ (2212) phase and impurities. (c) 2001 Elsevier Science B.V. All rights reserved.
\end{abstract}

Keywords: Synthesis of $\mathrm{Bi}_{2} \mathrm{Sr}_{2} \mathrm{Ca}_{2} \mathrm{Cu}_{3} \mathrm{O}_{10}$; Granular superconductivity; AC susceptibility; X-ray diffraction

\section{Introduction}

After the discovery of high- $T_{\mathrm{c}}$ superconductivity also in the $\mathrm{Bi}-\mathrm{Sr}-\mathrm{Ca}-\mathrm{Cu}-\mathrm{O}$ system (BSCCO) [1], it soon became apparent that such system actually consists of three different superconducting phases: $\mathrm{Bi}_{2} \mathrm{Sr}_{2} \mathrm{CuO}_{6+x}$ (2201 phase), $\mathrm{Bi}_{2} \mathrm{Sr}_{2} \mathrm{CaCu}_{2} \mathrm{O}_{8+x}$ (2212 phase) and $\mathrm{Bi}_{2} \mathrm{Sr}_{2} \mathrm{Ca}_{2} \mathrm{Cu}_{3} \mathrm{O}_{10+x}$ (2223 phase), with critical temperatures of about 20,80 and 110 $\mathrm{K}$, respectively [2].

The production of samples consisting only of the pure highest $T_{\mathrm{c}}$ phase has revealed to be not a

\footnotetext{
${ }^{*}$ Corresponding author. Tel.: +39-11-6707326; fax: +39-116691104.

E-mail address: truccato@to.infn.it (M. Truccato).
}

simple task, because of the large number of the variables involved in the process and of the greater thermodynamic stability of the 2212 phase with respect to the other ones. Several methods with different starting compounds (e.g. calcinated powders or glassy precursors) have been proposed either to obtain directly such samples in a bulk form [3-8] or to convert the 2212 phase samples into the 2223 phase [9-11].

Stoichiometric ratios between the precursors and details of the thermal treatments have widely proved to strongly influence the final 2223 phase content, as well as the chemical nature of the starting compounds $[8,12]$.

There have been in the past a few attempts to draw phase or time-temperature-transformation 
diagrams for the BSCCO system $[3,4,11,13,14]$, but a major problem has often been the evaluation of the relative 2212 to 2223 phase contents, due to their intrinsic mixing. The typical method for such evaluation is based on the relative intensities of the corresponding X-ray diffraction (XRD) peaks, but its precision is not very high, especially for very small phase contents. We have recently proposed a method [12] based on magnetic susceptibility measurements to demonstrate the influence of the starting chemical compounds on the 2223 phase growth; the present work is intended to validate this procedure by checking its capability to detect the influence of the annealing temperature.

\section{Sample preparation and characterisation}

Five samples have been prepared following exactly the same method, except for the final annealing temperature. Among several possibilities, commercial quality nitrate powders were chosen as the starting compounds because of the relatively good properties in the 2223 phase yield already shown in a previous study [12]. The nominal $\mathrm{Bi}: \mathrm{Pb}$ : $\mathrm{Sr}: \mathrm{Ca}: \mathrm{Cu}$ ratios have been fixed in 1.6:0.4:2:2:3, i.e. close to the ones commonly considered as a good starting point for the 2223 phase $[3,5,7,14]$. The powders were mixed, dissolved in water acidulated by nitric acid and then dried to obtain a simultaneous precipitation. The resulting deposits were calcinated at $500^{\circ} \mathrm{C}$ for $6-10 \mathrm{~h}$ and, after grinding and mixing, pressed at about $740 \mathrm{MPa}$ to form the pellets.

The thermal treatments consisted of annealing the five pellets for $120 \mathrm{~h}$ at the temperatures of $843^{\circ} \mathrm{C}, 846^{\circ} \mathrm{C}, 849^{\circ} \mathrm{C}, 852^{\circ} \mathrm{C}$ and $855^{\circ} \mathrm{C}$, respectively, with both heating and cooling rates of $5^{\circ} \mathrm{Cl}$ min. All the temperatures were measured at the sample position by means of the same tolerance class 2 type $\mathrm{K}$ thermocouple, so that the nominal accuracy of about $6^{\circ} \mathrm{C}$ corresponding to such temperatures can only introduce a possible homogeneous shift with respect to the International Temperature Scale ITS-90.

The pellets were subsequently cut in several pieces. One of them was subjected to the measurement of the XRD pattern on a SIEMENS D5000 diffractometer, equipped with Göbel mirrors, using Ni-filtered $\mathrm{CuK} \alpha$ radiation. The diffraction intensities in the $2 \theta$ range $3.5-50^{\circ}$ were collected in a $2 \theta-\theta$ scan mode, in steps of $0.02^{\circ}$ or $0.05^{\circ}$. The same part was later submitted to AC real $\left(\chi^{\prime}\right)$ and imaginary $\left(\chi^{\prime \prime}\right)$ susceptibility measurements from 80 to $120 \mathrm{~K}$ on a LAKESHORE 7225 system.

On another part of the pellet, 100-200 nm thick silver contacts were thermally evaporated in a vacuum chamber and joined to silver wires by means of a conducting silver paint. Such samples were used to measure the resistance vs. temperature characteristics by a standard four-probe technique.

\section{Experimental results and discussion}

Fig. 1 shows the resistance vs. temperature curves for the samples. It can be noted that each

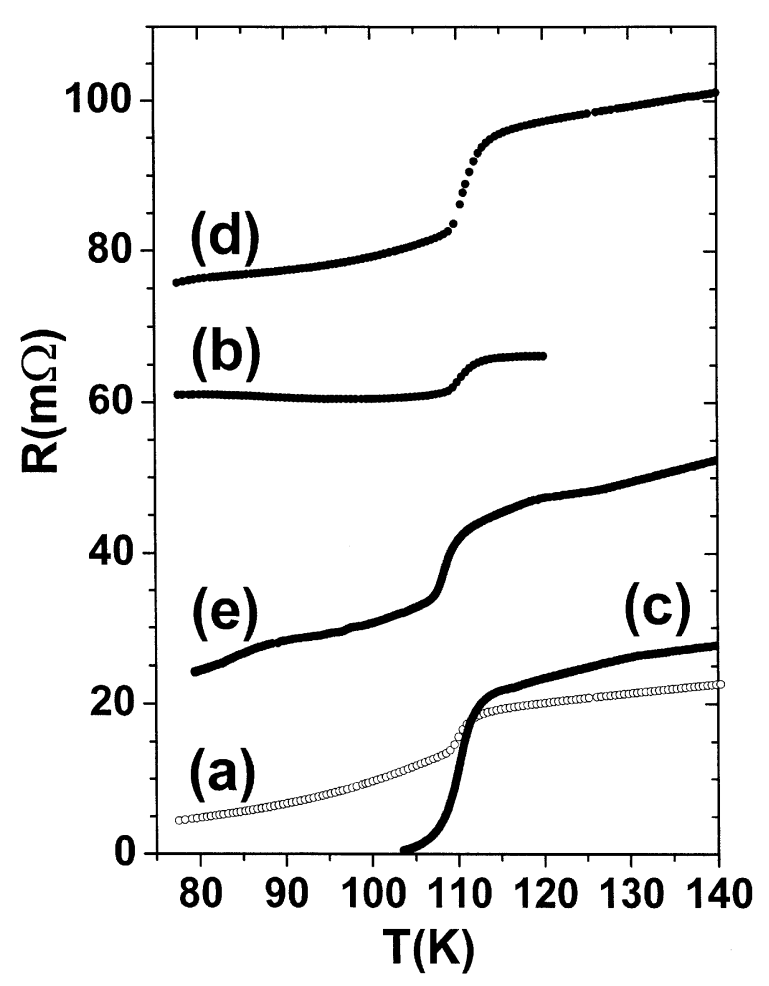

Fig. 1. Resistance vs. temperature curves for samples with annealing temperatures $843^{\circ} \mathrm{C}$ (a), $846^{\circ} \mathrm{C}$ (b), $849^{\circ} \mathrm{C}$ (c), $852^{\circ} \mathrm{C}$ (d) and $855^{\circ} \mathrm{C}(\mathrm{e})$. 
sample shows some drop in resistance at $T \approx 110$ $\mathrm{K}$, indicating that some amount of the 2223 phase is actually present. However, only in the case of the $849^{\circ} \mathrm{C}$ (Fig. 1c) annealing the zero resistance state is achieved, showing that a percolation path exists in the sample. This indicates that only in this case the percolation threshold volume fraction (which in the $3 \mathrm{D}$ case is about 0.16 ) is exceeded [15]. No other inference based on the resistance data can be drawn in a reliable way, i.e. consistently with the data exposed in what follows.

Fig. 2 represents the XRD patterns of the samples, where only the peaks corresponding to unambiguously identified phases have been labelled. It appears that 2212 is the dominant phase in all cases and also that the 2223 phase can always be detected as a minor phase, with a somewhat larger content in the $849^{\circ} \mathrm{C}$ sample (see, for example, the two peaks centred at about $2 \theta \approx 30.5^{\circ}$ and $2 \theta \approx 39.3^{\circ}$ ). Such an indication is consistent with the resistance measurements. About the recognised impurities, a temperature evolution can be observed. Starting from the lowest temperature, it can be seen that $\mathrm{Ca}_{2} \mathrm{PbO}_{4}$ is present only up to $849^{\circ} \mathrm{C}$, while $\mathrm{Ca}_{2} \mathrm{CuO}_{3}$ appears for the first time at $846^{\circ} \mathrm{C}$ and is still present at $855^{\circ} \mathrm{C}$. Moreover, some $\mathrm{SrPbO}_{3}$ content can be revealed for temperatures equal to or greater than $849^{\circ} \mathrm{C}$.

These results are in agreement with those reported previously in literature. The disappearance of $\mathrm{Ca}_{2} \mathrm{PbO}_{4}$ with increasing the temperature at a long enough sintering time $(\approx 20 \mathrm{~h})$ and the same $\mathrm{Pb}$ content has been observed both by Strobel et al. [13] and by Tatsuminago et al. [16]; moreover, the formation of a liquid phase due to the $\mathrm{Ca}_{2} \mathrm{PbO}_{4}$ decomposition at about $850^{\circ} \mathrm{C}$ has been reported by several authors $[3,8,14]$.

As far as $\mathrm{Ca}_{2} \mathrm{CuO}_{3}$ is concerned, its presence for annealing times not longer than $120 \mathrm{~h}$ has been reported only for temperatures equal to or greater than $850^{\circ} \mathrm{C}$, but it can be observed also at the temperature of $840^{\circ} \mathrm{C}$ if the annealing time is prolonged to $240 \mathrm{~h}[3,4,13]$. The reason for its presence in our samples could be twofold, in principle: on one hand, it could be interpreted as an incomplete synthesis of the 2223 phase by means of the reaction $1 / 2\left(\mathrm{CuO}+\mathrm{Ca}_{2} \mathrm{CuO}_{3}\right)+$ $2212 \rightarrow 2223$ [14]; on the other hand, it could re- sult from the decomposition reaction $4(2223) \rightarrow$ $4(2212)+2 \mathrm{Ca}_{2} \mathrm{CuO}_{3}+\mathrm{Cu}_{2} \mathrm{O}+1 / 2 \mathrm{O}_{2}$ that can take place because of an insufficient oxygen pressure $[17,18]$. From a general point of view, the evidence of an increasing amount of $\mathrm{Ca}_{2} \mathrm{CuO}_{3}$ with increasing the annealing temperature or duration seems to support the latter interpretation, and we agree with such hypothesis, arguing that the 2223 phase decomposition in our samples could result from a low local oxygen partial pressure in the microcavities which are formed in the bulk as the sintering proceeds.

In order to make possible a comparison with the magnetic susceptibility data which will be reported further on, a quantitative interpretation of the diffracted intensities has been attempted. It is well known that the integrated intensities of the diffraction peaks can be used to obtain the volume fractions corresponding to different phases in two or three component systems [19]. Actually, this method has already been implemented in several versions to measure the 2223 and 2212 phase volume fractions [14,20-22]. As our interest is mainly in the estimation of the 2223 phase content, such analysis has been performed only for the diffraction pattern showing the clearest peaks corresponding to this phase, that is the one shown in Fig. 2(c). The integrated intensities of the 2223 phase peaks and of the 2212 phase ones are reported in Tables 1 and 2, respectively. They have been calculated by fitting the diffraction pattern to the superimposition of gaussian peaks plus a background.

Following the method by Costa et al. [22], the peak intensities have been weighted by taking the relative intensities in the pure compounds given by the JCPDS-ICDD diffraction cards. Several diffraction cards have been examined both for peak identification and for intensity weighting of the two phases; in details the JCPDS-ICDD cards 41$374,42-415,42-743$ and 45-677 have been used for the 2223 phase, while the cards $41-317,41-932$, 42450, 45-676, 46-317 and 47-713 have been used for the 2212 phase [23]. It should be noted that the cards allow unambiguous identification of the peaks, giving always nearly the same position which is detected in our pattern, but, on the contrary, considerable differences in the relative in- 


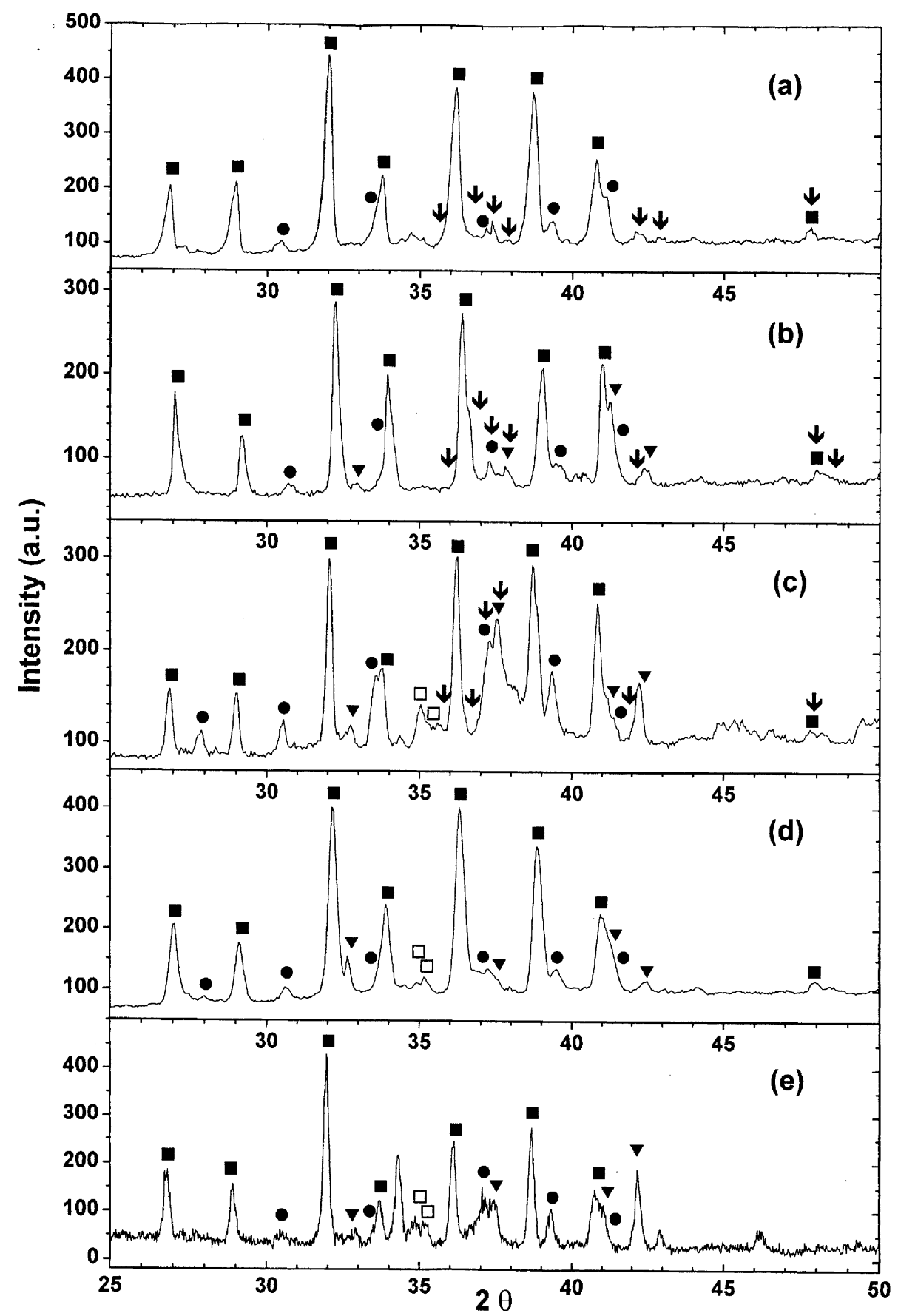

Fig. 2. XRD patterns for samples with annealing temperatures $843^{\circ} \mathrm{C} \mathrm{(a),} 846^{\circ} \mathrm{C} \mathrm{(b),} 849^{\circ} \mathrm{C}$ (c), $852^{\circ} \mathrm{C}$ (d) and $855^{\circ} \mathrm{C}$ (e). Solid squares label the 2212 phase lines, solid circles indicate the 2223 phase, arrows the $\mathrm{Ca}_{2} \mathrm{PbO}_{4}$, triangles the $\mathrm{Ca}_{2} \mathrm{CuO}_{3}$ and empty squares the $\mathrm{SrPbO}_{3}$. 
Table 1

Experimental integrated intensities $I^{p}$ for the 2223 phase peaks of Fig. 2(c), relative intensities $I^{\mathrm{t}}$ for the 2223 phase according to the JCPDS-ICDD card 41-374 and corresponding conversion factor $K_{2223}{ }^{\mathrm{a}}$. The average value for the conversion factor is $\bar{K}_{2223}=0.19$ with standard deviation $\sigma_{K_{2223}}=0.07$

\begin{tabular}{llccl}
\hline $2 \theta\left({ }^{\circ}\right)$ & $h k l$ & $I^{\mathrm{p}}$ (a.u.) & $I^{\mathrm{t}}(\%)$ & $K_{2223}$ \\
\hline 27.86 & $00 \underline{10}$ & 9.8 & 93 & 0.11 \\
30.52 & 115 & 7.2 & 33 & 0.22 \\
33.54 & $117+00 \underline{12}$ & 21.5 & 100 & 0.22 \\
38.94 & 200 & 5.4 & 21 & 0.26 \\
39.38 & $00 \underline{14}$ & 7.4 & 61 & 0.12 \\
\hline
\end{tabular}

${ }^{\mathrm{a}}$ The 117 and $00 \underline{12}$ peaks have not been disentangled due to insufficient angular resolution of the diffractometer.

Table 2

Experimental integrated intensities $I^{\mathrm{p}}$ for the 2212 phase peaks of Fig. 2(c), relative intensities $I^{t}$ for the 2212 phase according to the JCPDS-ICDD card 42-450 and corresponding conversion factor $K_{2212}$. The average value for the conversion factor is $\bar{K}_{2212}=0.62$ with standard deviation $\sigma_{K_{2212}}=0.17$

\begin{tabular}{lrccc}
\hline $2 \theta\left(^{\circ}\right)$ & $h k l$ & $I^{\mathrm{p}}($ a.u. $)$ & $I^{\mathrm{t}}(\%)$ & $K_{2212}$ \\
\hline 26.89 & 008 & 17.2 & 20 & 0.86 \\
29.01 & 113 & 15.3 & 33 & 0.46 \\
32.06 & 115 & 45.0 & 93 & 0.48 \\
33.79 & 0010 & 18.6 & 24 & 0.78 \\
36.21 & 117 & 49.1 & 69 & 0.71 \\
38.73 & 200 & 56.5 & 100 & 0.56 \\
39.32 & 202 & 9.3 & 20 & 0.46 \\
\hline
\end{tabular}

tensities can be observed among them. Therefore, the choice of the reference card for peak weighting is a crucial step which can considerably affect the final determination of the phase volumes. Indicating by $I^{\mathrm{p}}$ the integrated intensity of an experimental peak and by $I^{\text {t }}$ the relative intensity as resulting from a card, in an ideal situation they should be related by $I^{\mathrm{p}}=K I^{\mathrm{t}}$, where $K$ is a conversion factor between the two scales which is constant for all the peaks belonging to the same phase. If $K_{2212}$ indicates the conversion factor for the 2212 phase and $K_{2223}$ is the conversion factor for the 2223 phase, the ratio $K_{2223} /\left(K_{2212}+K_{2223}\right)$ corresponds to the 2223 phase volume fraction as usually determined by XRD data [14,20-22].

Unfortunately, the conversion factor $K$ resulting from $K=I^{\mathrm{p}} / I^{\mathrm{t}}$ for different peaks of the same phase is far from being constant. Therefore the $K$ values for all the peaks of each phase have been calculated by using all the relevant possible cards and both the average value $\bar{K}$ and the standard deviation $\sigma_{K}$ have been recorded in each case. Such values have subsequently been used to calculate the ratio $K_{2223} /\left(K_{2212}+K_{2223}\right)$ and the corresponding error: among all the possible choices for the reference cards of each phase, the combination delivering the minimum percentage error for the 2223 phase content was selected, i.e. the card 41374 for the 2223 phase and the card $42-450$ for the 2212 phase. The corresponding $I^{\mathrm{t}}$ and $K$ values are also reported in Tables 1 and 2, respectively. It turns out that for this choice the conversion factors are $\bar{K}_{2223}=0.19$ and $\bar{K}_{2212}=0.62$ and that the corresponding percentage errors are $\sigma_{K_{2223}} / \bar{K}_{2223}=$ $37 \%$ and $\sigma_{K_{2212}} / \bar{K}_{2212}=27 \%$ : the uncertainties are therefore quite large even in this most favorable case. Such values give the result $K_{2223} /\left(K_{2212}+\right.$ $\left.K_{2223}\right)=0.23 \pm 0.08$ for the 2223 phase content, corresponding to a $35 \%$ error.

It should be noted that such result is strongly dependent on the choice of the reference cards for peak weighting: the other possible card combinations give values of the 2223 phase volume fraction ranging from 0.18 to 0.41 with percentage errors up to $52 \%$.

Fig. 3 shows the results of the real part of the AC magnetic susceptibility $\chi^{\prime}$ vs. temperature measurements. All data have been taken at an applied magnetic field $H=200 \mathrm{~A} / \mathrm{m}$ with $f=1000$ $\mathrm{Hz}$ and are corrected by using an approximate demagnetisation factor corresponding to the sample geometry. The magnetic susceptibility was investigated only for temperatures greater than about $80 \mathrm{~K}$ to rule out contributions from the 2212 phase: it can be noted that actually no indication of a transition is observed in the region $T \approx 80 \mathrm{~K}$. On the other hand, a steep departure from zero is clearly detectable at $T \approx 110 \mathrm{~K}$ for all the samples, corresponding to the superconducting transition of the 2223 phase.

It has already been demonstrated in a previous work [12] that the magnetic behaviour of sintered samples made up of mixed 2212 and 2223 phases can be properly described by means of the effective medium model by Choy and Stoneham [24]. According to such model, samples can be thought of as consisting of superconducting spherical granu- 

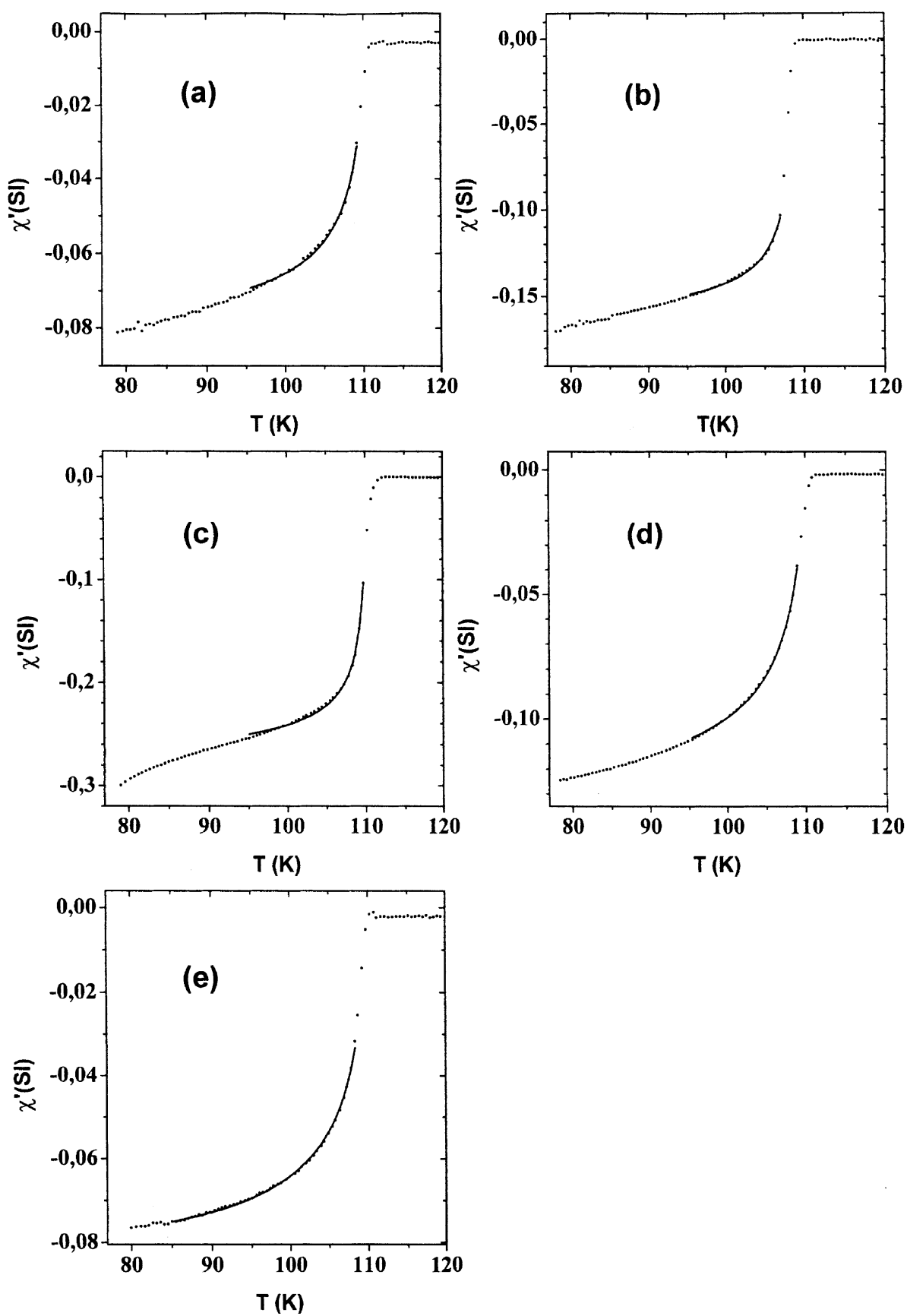

Fig. 3. Magnetic susceptibility vs. temperature curves for samples with annealing temperatures $843^{\circ} \mathrm{C}$ (a), $846^{\circ} \mathrm{C}(\mathrm{b}), 849^{\circ} \mathrm{C}(\mathrm{c}), 852^{\circ} \mathrm{C}$ (d) and $855^{\circ} \mathrm{C}$ (e). The measurements were performed in $H=200 \mathrm{~A} / \mathrm{m}$ at $f=1000 \mathrm{~Hz}$. Solid lines represent the fits to Eqs. (1) and (2).

lar inclusions of radius $a$ in a non-magnetic effective medium: the overall magnetic susceptibility is analytically calculated by taking into account the penetration of the magnetic field into the spheres for the typical penetration length $\lambda$. The final result can be expressed as: 


$$
\chi^{\prime}=-\frac{\frac{3}{2} c_{\mathrm{s}} Z_{\mathrm{L}}(a / \lambda)}{1-c_{\mathrm{s}}\left(1-Z_{\mathrm{L}}(a / \lambda)\right)},
$$

where $c_{\mathrm{s}}$ is the volume fraction of the superconducting grains and $Z_{\mathrm{L}}(x)=1-(3 / x)(\operatorname{coth}(x)-$ $1 / x)$. Assuming $c_{\mathrm{s}}$ to be constant, which, even in the case of a statistical distribution of the $T_{\mathrm{c}}$ 's among the grains, is a reasonable hypothesis for temperatures 1 or $2 \mathrm{~K}$ below the mean critical temperature, Eq. (1) can be used to describe the $\chi^{\prime}(T)$ behaviour if the temperature variation $\lambda(T)$ is known. Measurements both on polycrystalline tapes [25] and on single crystal whiskers [26] have shown that, at least in the $a b$ crystal planes, $\lambda(T)$ is well described for the 2223 phase near $T_{\mathrm{c}}$ by the BCS expression:

$\lambda(T)=\frac{\lambda(0)}{\sqrt{2\left(1-T / T_{\mathrm{c}}\right)}}$

with $\lambda(0) \approx 0.2 \mu \mathrm{m}$. Therefore, the theoretical law for the $\chi^{\prime}(T)$ behaviour can be obtained by inserting this experimental dependence into Eq. (1).

The applicability of this model to our samples deserves some discussion to examine whether its basic assumptions may be considered fullfilled or not.

The first assumption is that the shape of the granular inclusions is spherical: it is well known that real BSCCO samples consist of platelet-like grains with different dimensions, but, on the other hand, their random and isotropic orientation distribution can be considered to closely resemble spherical inclusions.

The second assumption is that samples consist only of two components, the spherical grains and the effective embedding medium, the latter being non-magnetic. It should be noted that such a two component description has already been widely adopted to describe the magnetic susceptibility of sintered high- $T_{\mathrm{c}}$ superconductors, even in the case of pure single-phase samples [26-30]. In these works, the grains are described as portions of highquality superconducting material with a large lower critical field $H_{\mathrm{cl}}$, while the embedding medium is identified with a matrix including both portions of poorly superconducting matter with a practically vanishing $H_{\mathrm{cl}}$, like for instance the grain boundaries, and voids. However, it is clear from XRD interpretation that our samples consist of a number of compounds. According to the single-phase description, both the 2212 and the 2223 phase should contribute with a highly superconducting grain component and with a poorly superconducting matrix component, but in the investigated temperature range both the 2212 phase components are in the normal non-diamagnetic state. On the other hand, as far as the 2223 phase is concerned, it has been shown for systems similar to ours $[29,30]$ that at about the applied field of $H=200 \mathrm{~A} / \mathrm{m}$ the superconductivity of the 2223 phase matrix component is strongly suppressed at least for $T>90 \mathrm{~K}$. Therefore, in order to apply the two component model to our samples, it is necessary to identify the spherical grains with the 2223 phase highly superconducting grain component, while the embedding medium consists of the 2223 phase poorly superconducting matrix component, both the 2212 phase components (matrix and grains) plus, obviously, the detected impurities and voids. The request for the embedding medium to be non magnetic is fullfilled by the fact that these superconducting components are in the normal state.

The third hypothesis of the model is that the spherical grains are in the Meissner state, so that the magnetic flux is not trapped inside the grains in the form of isolated vortexes but can only penetrate from the surface in a continuous mode. The discussion of such hypothesis requires knowledge of the lower critical field $H_{\mathrm{cl}}$. Recent DC magnetisation measurements performed on 2223 phase whisker-like crystals have given $H_{\mathrm{cl}}(0)=135 \mathrm{G}$ [26], which is in general agreement with but more reliable than similar determinations on polycrystalline samples, so that this value can reasonably be taken as the lower critical field for the grains. Assuming the empirical formula $H_{\mathrm{cl}}=H_{\mathrm{cl}}(0)[1-$ $\left.\left(T / T_{\mathrm{c}}\right)^{2}\right]$ to hold, this implies that at the applied field of $H=200 \mathrm{~A} / \mathrm{m}$ the grains should remain in the Meissner state up to about $T=109 \mathrm{~K}$.

The fourth assumption is that the grains can be considered isolated, in the sense that each of them interacts only with the external applied field $H$, neglecting the local perturbations induced by the magnetic field excluded from the neighbouring 
grains. Obviously, in real samples grains are not isolated, however the experimental results by Campbell et al. [31] for closely packed grain samples indicate that no significant error should be introduced by possible grain couplings.

Such considerations show that in our experimental conditions all the hypothesis of the ChoyStoneham model are expected to hold in the temperature range between approximately $90 \mathrm{~K}$ and $109 \mathrm{~K}$.

The fits of the model represented by Eqs. (1) and (2) to the experimental data are shown as solid lines in Fig. 3. Due to the statistical distribution of the grain sizes, $a$ has been considered a parameter in the fits just like $c_{\mathrm{s}}$, which was the principal quantity to be measured. A small variation of $T_{\mathrm{c}}$ in a range of $0.3 \mathrm{~K}$ around $T_{\mathrm{c}}=110 \mathrm{~K}$ was also permitted to optimise the fits. No other degree of freedom was allowed for the fits. It can be noted that the agreement with the experiment is very good in the temperature range $95 \mathrm{~K}<T<107 \mathrm{~K}$ for all samples. In the region $T<95 \mathrm{~K}$ the model has a general tendency to underestimate the real $\chi^{\prime}$ values, since the theoretical law saturates while experimental data still decrease. This effect appears to be sample dependent and can be ascribed to the quality of the 2223 phase matrix, as will be further discussed below.

The results of the fits are reported in Table 3. It can be observed that all the samples show a 2223 phase volume fraction $c_{\mathrm{s}}$ below the percolation threshold 0.16 , except the one corresponding to the $849^{\circ} \mathrm{C}$ annealing temperature, in agreement with the resistivity results. About the $a$ values, it is worth noting that the corresponding spherical volumes appear to be consistent with the grain

Table 3

2223 phase grain volume fraction $c_{\mathrm{s}}$, average radius $a$ and numerical density $n$ of the grains (see text) deduced from the fits of the $\chi^{\prime}$ experimental data to Eqs. (1) and (2)

\begin{tabular}{llll}
\hline $\begin{array}{l}\text { Annealing } \\
\text { temp. }\left({ }^{\circ} \mathrm{C}\right)\end{array}$ & $c_{\mathrm{s}}$ & $a(\mu \mathrm{m})$ & $n\left(\mathrm{~mm}^{-3}\right)$ \\
\hline 843 & $0.061 \pm 0.002$ & $4.1 \pm 0.3$ & $(20 \pm 4) \times 10^{4}$ \\
846 & $0.117 \pm 0.002$ & $6.6 \pm 0.4$ & $(10 \pm 2) \times 10^{4}$ \\
849 & $0.195 \pm 0.003$ & $6.2 \pm 0.3$ & $(20 \pm 3) \times 10^{4}$ \\
852 & $0.101 \pm 0.002$ & $3.3 \pm 0.1$ & $(69 \pm 6) \times 10^{4}$ \\
855 & $0.064 \pm 0.001$ & $3.5 \pm 0.1$ & $(37 \pm 5) \times 10^{4}$ \\
\hline
\end{tabular}

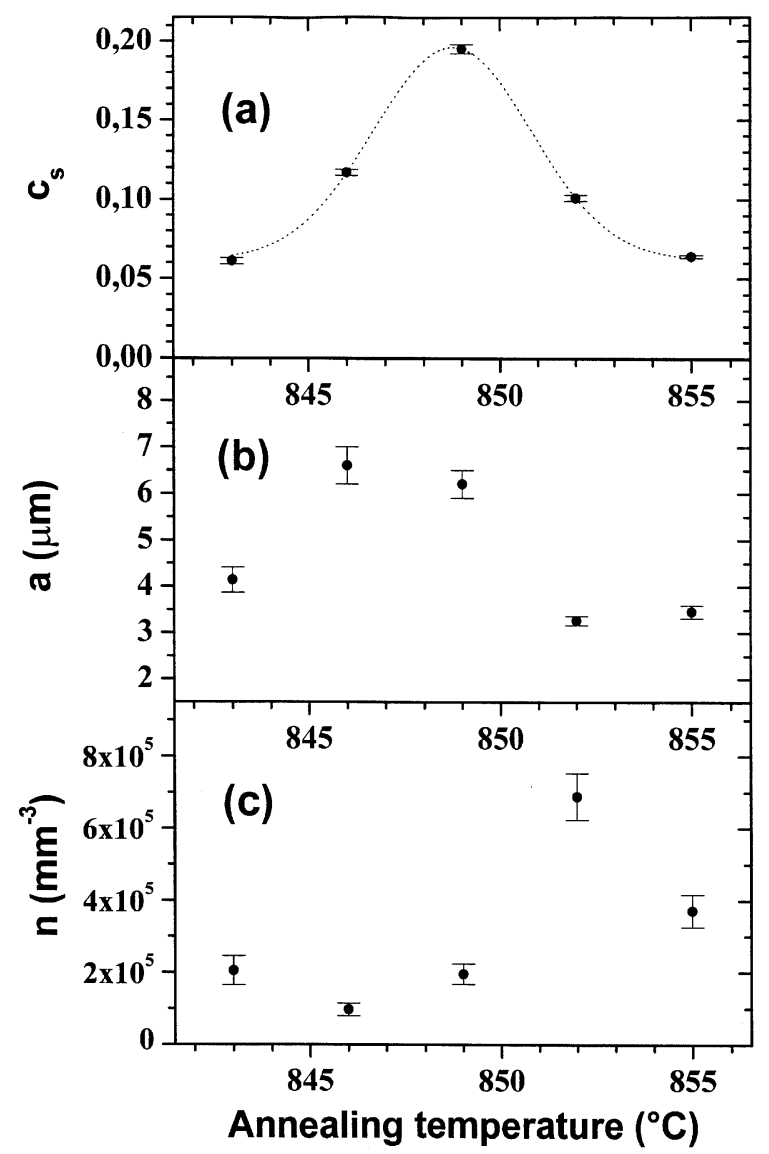

Fig. 4. Temperature evolution of the 2223 phase volume fraction $c_{\mathrm{s}}$ (a), of the average radius $a$ (b) and of the numerical density $n$ of the grains (c). The dotted curve serves only as a guide for the eye.

volumes obtained from direct measurement of the grain sizes at the SEM, so that the spherical inclusion assumption is confirmed.

Moreover, a temperature evolution can be detected both for $c_{\mathrm{s}}$ and $a$, as graphically highlighted in Figs. 4(a) and (b). The largest 2223 phase content in our system seems to be obtained for $T=$ $849^{\circ} \mathrm{C}$ and sharply decreases in both directions for a temperature change of only $3^{\circ} \mathrm{C}$. This is consistent with previous results indicating $T=850$ $855^{\circ} \mathrm{C}$ as the temperature required to maximise the 2223 phase content in similar systems [14,20]. An analogous temperature dependence can be seen also for the grain radius $a$, with the highest radii 
for $T=846^{\circ} \mathrm{C}$ and $849^{\circ} \mathrm{C}$ and some evidence that annealing temperatures higher than $T=849^{\circ} \mathrm{C}$ induce smaller 2223 phase grain volumes.

The information about $c_{\mathrm{s}}$ and $a$ can be combined to obtain the numerical density of grains $n$ in the spherical approximation through the relation $n=3 c_{\mathrm{s}} / 4 \pi a^{3}$. The corresponding results are also listed in Table 3 and displayed in Fig. 4(c), where the interesting observation can be made that the number of grains seems to increase for $T>849^{\circ} \mathrm{C}$.

It should be stressed that the values and the errors obtained for $c_{\mathrm{s}}$ and $a$ are quite robust with respect to variations of the fit details like the temperature range and, to a certain extent, the demagnetising factor: therefore the average percentage errors of $2 \%$ and $4 \%$ can be regarded as the typical precision that can be achieved by this method in measuring $c_{\mathrm{s}}$ and $a$, respectively. The major problem with the model seems to occur generally at $T<95 \mathrm{~K}$ where a stronger diamagnetic signal than predicted is detected. This deviation is not steep enough to be due to the onset of the 2212 phase superconducting transition, but could be ascribed to an additional contribution coming from the 2223 phase matrix component, which at the applied field $H=200 \mathrm{~A} / \mathrm{m}$ should become superconducting by decreasing the temperature, if its lower critical field $H_{\mathrm{cl}}(0)$ is large enough. Such interpretation can be confirmed by considering Fig. 3(c), which shows that the $\chi^{\prime}$ behaviour for the sample with the maximum 2223 phase grain content is the one where the departure from the model at $T<95 \mathrm{~K}$ is most apparent. It is reasonable to suppose that, for this sample, also the 2223 phase matrix has better superconducting properties. Such a problem could in principle be solved by increasing the applied magnetic field to suppress the 2223 phase matrix superconductivity, but caution should be used because this would also reduce the validity range of the fits on the high temperature side by breaking down the Meissner state for the 2223 phase grains in a wider range near $T_{\mathrm{c}}$.

The information of Table 3 can also be exploited in another way. It is clear from the $c_{\mathrm{s}}$ values that the embedding medium around the highly superconducting grains of the 2223 phase is always dominant in volume. According to our in- terpretation, this medium consists of the poorly superconducting 2223 phase matrix component, the 2212 phase, impurities and voids, but, on one hand, it is apparent from X-ray diffraction patterns in Fig. 2 that the impurities are generally a small fraction with respect to the 2212 and 2223 components and, on the other hand, SEM observations suggest that on the average the voids play a more important role than impurities in our samples. We can therefore adopt a simplified description in which the embedding medium is thought to consist only of the 2212 phase and voids, neglecting the contribution both of the impurities and of the 2223 phase matrix, the latter being expected to be only a small fraction of the highly superconducting 2223 phase grain volume fraction $c_{\mathrm{s}}$. The corresponding volume fractions $c_{2212}$ and $c_{\text {void }}$ can thus be estimated by using the experimental sample densities $\rho$ and the ideal densities of the 2223 and of the 2212 phase $\left(\rho_{2223}=\right.$ $6.26 \mathrm{~g} / \mathrm{cm}^{3}$ and $\rho_{2212}=6.52 \mathrm{~g} / \mathrm{cm}^{3}$, respectively) via the equations $c_{\mathrm{s}}+c_{2212}+c_{\text {void }}=1$ and $\rho=$ $c_{\mathrm{s}} \rho_{2223}+c_{2212} \rho_{2212}$. The resulting values are reported in Table 4 . It should be noted that the $c_{\text {void }}$ values are quite similar in all the samples and turn out to be always greater than the corresponding $c_{\mathrm{s}}$ values, so that the presence of voids cannot be neglected in such systems. This is probably the most important difference between our measurement of the 2223 phase volume fraction and the usual XRD methods [14,20-22]. X-rays by themselves are unable to detect voids and what in these papers is indicated as the 2223 phase volume fraction is actually, in our notation, the ratio

Table 4

Sample density $\rho, 2212$ phase volume fraction $c_{2212}$, void volume fraction $c_{\text {void }}$ and ratio $c_{\mathrm{s}} /\left(c_{\mathrm{s}}+c_{2212}\right)$ as resulting from the simplified embedding medium description discussed in the text and from $c_{\mathrm{s}}$ data of Table 3

\begin{tabular}{lllll}
\hline $\begin{array}{l}\text { Annealing } \\
\text { temp. }\left({ }^{\circ} \mathrm{C}\right)\end{array}$ & $\rho\left(\mathrm{g} / \mathrm{cm}^{3}\right)$ & $c_{2212}$ & $c_{\text {void }}$ & $c_{\mathrm{s}} /\left(c_{\mathrm{s}}+c_{2212}\right)$ \\
\hline 843 & 4.75 & 0.67 & 0.27 & 0.08 \\
846 & 5.08 & 0.67 & 0.22 & 0.15 \\
849 & 5.07 & 0.59 & 0.21 & 0.25 \\
852 & 4.44 & 0.58 & 0.31 & 0.15 \\
855 & 5.13 & 0.73 & 0.21 & 0.08 \\
\hline
\end{tabular}


$c_{\mathrm{s}} /\left(c_{\mathrm{s}}+c_{2212}\right)$. A part from the better precision of the magnetic method, this different approach to the problem leads the X-ray method to overestimate the 2223 phase content due to the assumption of a vanishing $c_{\text {void }}$ in the denominator. For comparison purposes, also the ratios $c_{\mathrm{s}} /\left(c_{\mathrm{s}}+c_{2212}\right)$ have been reported in Table 4 , which should correspond to the 2223 phase volume fractions as calculated from XRD peak intensities.

It can be noted that the 0.25 value for the $849^{\circ} \mathrm{C}$ sample is in good agreement with the $0.23 \pm 0.08$ value resulting from the XRD analysis of Fig. 2(c), confirming previous considerations on the methods.

As a final remark, it can be observed that the highest void fraction is achieved for the annealing temperature $T=852^{\circ} \mathrm{C}$, which is also the temperature giving the highest numerical density of grains $n$. This fact seems indeed to support the idea that with increasing the annealing temperature the external part of the grains undergoes the decomposition $4(2223) \rightarrow 4(2212)+2 \mathrm{Ca}_{2} \mathrm{CuO}_{3}+$ $\mathrm{Cu}_{2} \mathrm{O}+1 / 2 \mathrm{O}_{2}$ to restore the equilibrium oxygen partial pressure in the sample microcavities. Such a process would likely dissolve the thinnest regions of the grains and therefore induce both grain splitting and production of a gaseous phase which should result in a higher void fraction.

\section{Conclusions}

In the present study, we have investigated the dependence of the 2223 phase growth on the annealing temperature by applying an effective medium model to the experimental magnetic susceptibility data. The data are well explained by the model in the temperaure range $95 \mathrm{~K}<T<$ $107 \mathrm{~K}$ at the magnetic field $H=200 \mathrm{~A} / \mathrm{m}$ and both the 2223 phase volume fraction and the medium grain radius have been determined.

It has also been shown that, for a fixed annealing time of $120 \mathrm{~h}$, the annealing temperature corresponding to the highest 2223 phase content is $T=849^{\circ} \mathrm{C}$, which is in agreement with previous results.

We have also given evidence that a 2223 phase decomposition can take place in the bulk of the samples within $120 \mathrm{~h}$ for high enough annealing temperatures.

Finally, the different characteristics in measuring the 2223 phase content have been briefly compared between our method and the XRD one.

\section{Acknowledgements}

The authors wish to acknowledge Prof. Mezzetti and co-workers for access and assistance at the susceptibility measurements. We are also grateful to Prof. G. Chiari for allowing access to the X-ray diffractometer and to Dr. M. Prencipe for useful discussion on the interpretation of XRD patterns. Special thanks to Ms. N. Rizzi and Dr. S. Sanguinetti for their valuable help during the first part of the work.

\section{References}

[1] C. Michel, M. Hervieu, M.M. Borel, A. Grandin, F. Deslandes, J. Provost, B. Raveau, Z. Phys. B 68 (1987) 421.

[2] H. Maeda, Y. Tanaka, M. Fukitoki, T. Asano, Jpn. J. Appl. Phys. Lett. 27 (1988) L209.

[3] Y. Massalker, A.N. Sembira, J. Baram, J. Mater. Res. 8 (10) (1993) 2445.

[4] D. Shi, M. Tang, M.S. Boley, M. Hash, K. Vandervoort, H. Claus, Y.N. Lwin, Phys. Rev. B 40 (4) (1989) 2247.

[5] E. Chavira, R. Escudero, D. Rios-Jara, M. Leon, Phys. Rev. B 38 (13) (1988) 9272.

[6] R. Cloots, A. Rulmont, P.A. Godelaine, H.W. Vanderschveren, H. Bowgrine, M. Ausloos, Europhys. Lett. 30 (8) (1995) 487.

[7] B.W. Veal, H. Claus, J.W. Downey, A.P. Paulikas, K.G. Vandervoort, J.S. Pan, D.J. Lam, Physica C 156 (1988) 635.

[8] R. Cloots, B. Bougrine, M. Houssa, S. Stassen, L. D’Urzo, A. Rulmont, M. Ausloos, Physica C 231 (1994) 259.

[9] I. Matsubara, H. Tanigawa, T. Ogura, H. Yamashita, M. Kinoshita, T. Kawai, Appl. Phys. Lett. 57 (23) (1990) 2490.

[10] I. Matsubara, H. Tanigawa, T. Ogura, H. Yamashita, M. Kinoshita, T. Kawai, Appl. Phys. Lett. 58 (4) (1991) 409.

[11] I. Matsubara, R. Funahashi, T. Ogura, H. Yamashita, Y. Uzawa, K. Tanizoe, T. Kawai, Physica C 218 (1993) 181.

[12] C. Manfredotti, M. Truccato, P. Volpe, P. Benzi, N. Rizzi, S. Sanguinetti, D. Allasia, Physica C 303 (1998) 94.

[13] P. Strobel, T. Fournier, J. Less-Comm. Met. 164-165 (1990) 519.

[14] S. Nhien, G. Desgardin, Physica C 272 (1996) 309. 
[15] R. Zallen, The Physics of Amorphous Solids, Wiley, New York, 1983, p. 186.

[16] M. Tatsumisago, S. Tsuboi, N. Tohge, T. Minami, Appl. Phys. Lett. 57 (2) (1990) 195.

[17] L.M. Rubin, T.P. Orlando, J.B. Vander Sande, G. Gorman, R. Savoy, R. Swope, R. Beyers, Appl. Phys. Lett. 61 (16) (1992) 1977.

[18] L.M. Rubin, T.P. Orlando, J.B. Vander Sande, G. Gorman, R. Savoy, R. Swope, R. Beyers, Physica C 217 (1993) 227.

[19] B.D. Cullity, Elements of X-ray Diffraction, AddisonWesley, Reading, MA, 1978, p. 411.

[20] X.H. Gao, D. Gao, J.H. Li, J. Li, S.F. Jiang, Physica C 229 (1994) 124.

[21] X.H. Gao, J. Li, S.F. Jiang, D. Gao, G.D. Zheng, S. Gao, Physica C 244 (1995) 321.

[22] F.M. Costa, R.F. Silva, J.M. Vieira, Physica C 323 (1999) 23.

[23] X-ray Powder Diffraction File, cards 41-317, 41-374, 41$932,42-415,42-450,42-743,45-676,45-677,46-317$ and $47-$
713, JCPDS-International Center for Diffraction Data, Newtown Square, PA, USA.

[24] T.C. Choy, A.M. Stoneham, J. Phys.: Condens. Mat. 2 (1990) 939.

[25] Q. Li, M. Suenaga, J. Gohng, D.K. Finnemore, T. Hikata, K. Sato, Phys. Rev. B 46 (5) (1992) 3195.

[26] I. Matsubara, R. Funahashi, K. Ueno, H. Yamashita, T. Kawai, Physica C 256 (1996) 33.

[27] D.X. Chen, J. Nogues, K.V. Rao, Cryogenics 29 (1989) 800.

[28] D.X. Chen, A. Sanchez, T. Puig, L.M. Martinez, J.S. Munoz, Physica C 168 (1989) 652.

[29] D.X. Chen, Y. Mei, H.L. Luo, Physica C 167 (1990) 317.

[30] R.B. Goldfarb, M. Lelental, C.A. Thompson, in: R.A. Hein, T.L. Francavilla, D.H. Liebenberg (Eds.), Magnetic Susceptibility of Superconductors and Other Spin Systems, Plenum Press, New York, 1992.

[31] A.M. Campbell, F.J. Blunt, J.D. Johnson, P.A. Freeman, Cryogenics 31 (1991) 732. 